\title{
First Survey Results of Quantified User Behavior in User-in-the-Loop Scenarios for Sustainable Wireless Networks
}

\author{
Rainer Schoenen*†, Gurhan Bulu ${ }^{\S}$, Amir Mirtaheri*, Tamer Beitelmal*, Halim Yanikomeroglu* \\ * Department of Systems and Computer Engineering, Carleton University, Ottawa, Ontario, Canada \\ $\dagger$ Communication Networks (ComNets), Faculty 6, RWTH Aachen University, Germany \\ $\S$ Department of Electrical \& Electronics Engineering, Hacettepe University, Turkey \\ e-mail: \{rs, bulu, halim\}@sce.carleton.ca
}

\begin{abstract}
Traffic in mobile radio networks is expected to continue to increase by $100 \%$ per year. This imposes a big challenge for future generations (4G and 5G) of access technologies which were previously dimensioned for over-provisioning especially in the busy hours. Recent forecasts hint that this assumption and approach will not be tractable anymore. Instead the demand will exceed the supply more and more frequently causing congestion not only in short term but over longer periods of time. Any approach to engineer networks greener by better spectral efficiency only will fail to meet the global objective, if demand keeps on increasing faster than supply and efficiency.

Instead, the User-in-the-loop approach allows to shape the demand where it originates either in space or time. This is achieved by incentives for users to change location or by dynamic tariffs to shift the use of congested resources out of the busy hours. This works as the smart grid of communications.

While some work has already been done in this new field, the user behavior to the controlled demand shaping was based on assumptions. In this paper, the assumptions were confirmed by recent survey results which indicate that shaping user behavior works sufficiently well. Models of the user response are given and analysis and simulation results show the advantages and gains of this approach.
\end{abstract}

Index Terms-User-in-the-loop (UIL); IMT-Advanced; LTE, relays; demand shaping; tariffs; spatial and temporal control

\section{INTRODUCTION}

D EMAND for carrying higher and higher data rates in the next 10 years is clearly foreseeable [1]-[4]. The rise of smart phones and laptop dongles will bring traffic increases of $100 \%$ per year (Fig. 2) - a continuing trend in the last 5 years. Cellular wireless networks are traditionally oversized in order to carry all traffic, but this becomes harder even with $4 \mathrm{G}$ and $5 \mathrm{G}$ and can never keep up with demand at this increase rate [5]. Meanwhile energy consumption becomes more important and going green is a serious concern. Unfortunately every increase of capacity, in the best case assumed without increase of power consumption, will be eaten up by the exponential rise of traffic demand. Thus the traditional over-provisioning approach requires to spend even more CAPEX and power for denser cells, e.g., pico and femtocells.

Certainly the evolution of IMT-Advanced systems [6] will continue. However, there is an orthogonal approach which

Gurhan Bulu is supported under postdoctoral research fund by The Scientific and Technological Research Council of Turkey (TUBITAK). doesn't require more CAPEX and power and can be used in combination with already existing techniques. This User-inthe-loop (UIL) concept is able to boost the spectral efficiency by substantial amounts [7]. The spatial UIL method works by convincing users to change their location to a better one [8], similar to what people are used to do in WiFi networks. The temporal UIL method works by fully dynamic tariffs [9] which shape the user demand in times of congestion. This works well in line with experts favoring usage-based pricing [10]-[12]. Also, it appears more logical in times of economic and ecologic uncertainties that exponential increase must be questioned when it comes with a negative impact for society or our ecosystem.

In technical terms, UIL aims at stabilizing the demand to any sustainable level. In the case of cellular networks, it helps keeping traffic below the capacity at all times [9]. Other promising techniques to improve the supply side, e.g., decodeand-forward relays [13], can be applied independently.

User-in-the-loop (Figure 1) works by having the system including the user, wireless equipment and channel in a control loop. The controller knows the system state, especially the traffic load condition and channel conditions (pathloss) for all locations in the cell and derives a decision to influence the user based on this state. The decision is a set of information and incentives, in a user friendly way, to convince users to either move, postpone or abandon their session request in case of congestion. The control can range from just informational to convincing with a monotonic force depending on the severity of the situation.

In previous papers the user behavior was based on assumptions [8], [9], but this paper contributes the very first survey results to allow quantitative modeling. This survey A sample population of Summer 2011 was 60 university students in Canada. Although not perfectly representative, this is the first result of its kind and serves as an indicator of what is the potential and principal behavior of users in a system-theoretic framework. A larger sample is possible at a later point based on the methodology presented here.

The paper is organized as follows: The first sections define the spatial and temporal UIL model. Then the survey results give quantitative answers on how to model the user behavior. The last section shows performance results achieved in scenarios based on the IMT-Advanced evaluation [14]. 


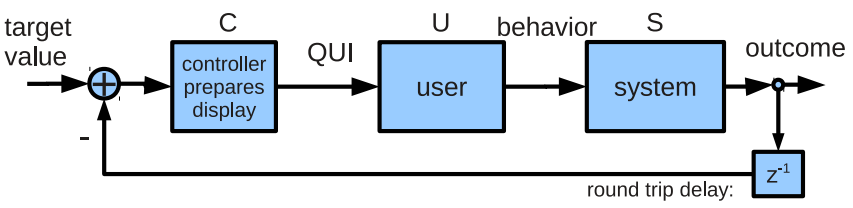

Fig. 1. User-in-the-loop allows closed-loop control of user and system [8]

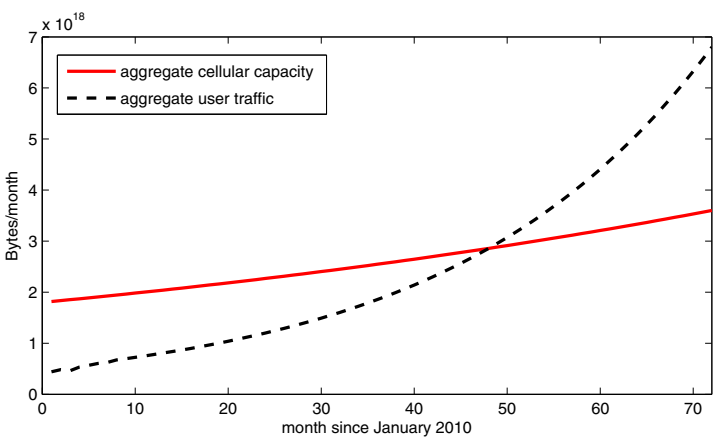

Fig. 2. Aggregate mobile traffic prediction $r(t)$ and capacity limitation.

\section{Spatial UiL CONTROL}

Figure 1 shows the general UIL concept. The controller provides suitable information to the user and the user should be convinced to change his location voluntarily from his current location $\overrightarrow{p_{1}}=\left(x_{1}, y_{1}\right)$ to $\overrightarrow{p_{2}}$. The network controller knows the current signal quality $\sigma\left(\overrightarrow{p_{1}}\right)$ (SINR-based) or the spectral efficiency $\gamma\left(\overrightarrow{p_{1}}\right)$ from UT measurements, and the expected level $\gamma\left(\overrightarrow{p_{2}}\right)$ from a database of measurements of all UTs at all locations in the past. The user knows his utility advantage of $\Delta u_{1,2}=u\left(\overrightarrow{p_{2}}\right)-u\left(\overrightarrow{p_{1}}\right)$ when doing the move. This utility $u$ can be either financial (discount for voice calls during busy hours) or an increased data rate (for best effort data traffic). The network provides the information in which direction or to which location to move by the gradient $-\nabla \sigma(\vec{p})$ of the potential field at position $\overrightarrow{p_{1}}$. The user should have all information to make his decision. The user can see in which direction to move best and how far $d_{1,2}=\left|\overrightarrow{p_{2}}-\overrightarrow{p_{1}}\right|$ the next improvement step is. A fraction $p_{M}$ of users actually participates in moving, the remaining $\left(1-p_{M}\right)$ stay in place. It accounts for all users that cannot move, do not want to move, or have no sufficient incentive to move. Previously this value was assumed constant [8], but in this paper it depends on the incentive to the user and the proposed $d_{1,2}$. The survey results in section IV quantify exactly this value. The output of the user block (Figure 1) is the new location $\overrightarrow{p_{2}}$. It is described by a Bernoulli random process where $p_{M}$ is the probability of a move from $\overrightarrow{p_{1}}$ to $\overrightarrow{p_{2}}$ for $d_{1,2}$ meters and $\left(1-p_{M}\right)$ of no movement at all. It depends on the incentive utility $u$ and proposed distance $d_{1,2}$. The survey results in section IV provide all the information needed. The target value $\gamma_{\Theta}\left(M I_{\min }\right)$ is the least $\gamma$ this UT should achieve after the movement. It is set by the operator. Half of the maximum or $2.5 \mathrm{bit} / \mathrm{s} / \mathrm{Hz}$ is a good operating point. It is possible to have multiple levels for $\gamma_{\Theta, i}$ and corresponding incentives.

\section{TEMPORAL UIL CONTROL}

Flat-rate pricing is the cause of heavy-tailed traffic distributions [9] and unbounded exponential demand increase. Thus usage-based pricing is favored in the pricing literature [10][12]. For UIL, a fully dynamic usage-based pricing is assumed [9] with prices known and displayed openly to the

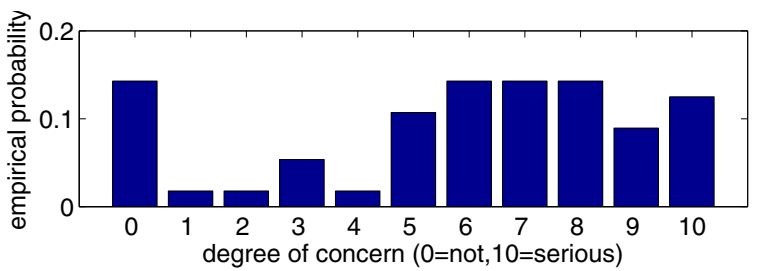

Fig. 3. Your wireless data usage has a carbon footprint proportional to your use. If you are told that for each MB that you download, you produce around 30 grams of $\mathrm{CO}_{2}$ [9] (i.e. about 17 liters of carbon dioxide), on a scale of 0 to 10 (with 10 being the most concerned) how much do you care to adjust your data usage to be greener?

user in advance. The pricing scheme will change the user behavior as modeled here. The principle is clear - the user will use less traffic when the price goes up. Previously a linear model with constant elasticity has been assumed [9], but here the survey results allow modeling the detailed user behavior. The control ratio $p$ is defined as the quotient of the controlled output rate $r_{c}$ divided by the uncontrolled output rate $r_{u}$. It can alternatively be interpreted as the proportion of users that stay with their original demand, while $1-p$ of the users change their mind and do not trigger the data transmission.

The traffic demand is controlled (shaped) according to the control model of Figure 1. For each time step $t$ (by month or day here) the controller knows the error $\epsilon(t)=$ $R_{\text {target }}(t)-r(t)$. In order to reduce the uncontrolled traffic load $r_{u}$ to $R_{\text {target }}$, the control ratio $p$ must be chosen as $p=R_{\text {target }} / r$. The next step is the adaptation of a pricing parameter, depending on the pricing model. If we assume proportional pricing with one free parameter, then $b$ is adapted taking the inverse of the user response function (from Section IV). This closes the control loop, because the tariff model and price information are the input to the user block. The user acts according to his known stochastic behavior and the target value is achieved.

\section{SURVEY RESUlTS}

This very first survey has been conducted in summer 2011 among 60 university students. The questions were all variations of this type: "For mobile service $S$ assume you have options to move $d$ meters or postpone for $T$ minutes and get an incentive $I$ to do so, how much $d$ or $T$ would be acceptable?". One example question is "Assume that you are walking in downtown and you have to make a phone call via your cell phone. The duration of your call is expected to be between 5 to 10 minutes. Assume that your cell phone company charges you $\$ 0.50$ per minute. However, it also offers you discount if you are willing to change your location. In the following table, choose the maximum distance you are willing to walk in return for the stated amount of offered discount". Figure 3 shows the concern about the wireless carbon footprint which might motivate a UIL move even without explicit incentive. But for the model so far it is assumed that zero incentive results in zero change from uncontrolled use of mobile services. In this paper, results are displayed for each service and incentive and in two ways: The upper graphs show the empirical CCDF for the acceptable movement distance $d$ and the fraction $p_{M}$ of users conforming, given the incentive as parameter. The lower graphs show the PMF distribution of the acceptable UIL reaction (move or delay) and its average, with the incentive on the $\mathrm{x}$-axis. Figure 4 shows the results for voice and video service. The results show a remarkable elasticity, i.e., 


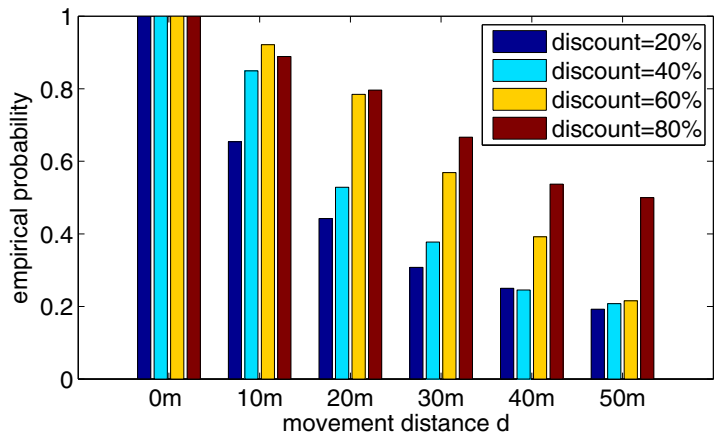

(a) Empirical CCDF for voice calls: With a given discount and a pote distance $\mathrm{d}$ to move, how likely $\left(p_{M}\right)$ is the movement of the user.

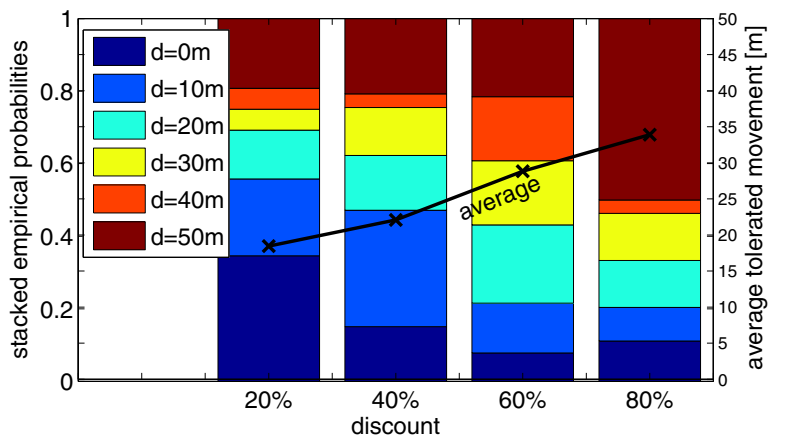

(c) For a given discount incentive for voice calls, what is the tolerated movement distribution among all users and its average.

Fig. 4. Voice and video service comparison with movement control and discount incentives.

(d) For a given discount incentive for video streaming, what is the tolerated movement distribution among all users and its average.

(b) Empirical CCDF for video streaming: With a given discount and a potential distance $\mathrm{d}$ to move, how likely $\left(p_{M}\right)$ is the movement.

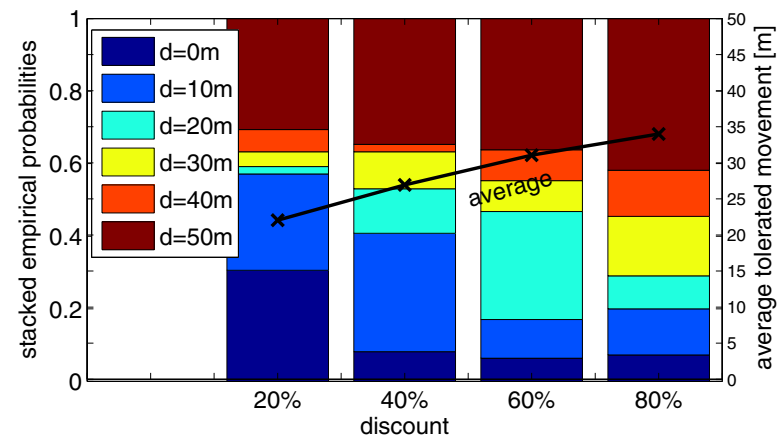

TABLE I

IMT-ADVANCED SCENARIO SPECIFICATIONS

acceptance of the proposed relocation, with video being more elastic (perceived as less important service). Figure 5 also shows voice and video service, but with a proposed deferral. The results indicate that there is a significant acceptance. This is sufficient to relief busy-hour congestion [9]. Especially the UIL control of video streaming (with 100 times higher rate requirements) is tolerated easily. Not shown here are the reactions to abandon or offload the demand to fixed line access at home. For data services, i.e., browsing and app downloading, the results are shown in Figure 6. The speedup incentive turns out to be very convincing. It comes naturally with a resource proportional scheduler so there is low effort in implementing this incentive.

\section{Simulation Results}

The system model of Fig. 1 is now applied to a cellular scenario. The quantitative user response is assumed as surveyed, i.e., the probability $p_{M}$ to participate depends on the proposed distances to the next better location. In this paper we assume the IMT-Advanced system model [14]. Table I provides the data for the scenarios taken into account. They are representative for the whole range between densely populated (UMi) to countryside setups (RMa). At the cell borders SINR is close to $0 \mathrm{~dB}$ with high fluctuations. Over the cell area $\operatorname{SINR}(\sigma)$ results are obtained by numeric analysis and are translated to spectral efficiency $\gamma$ in bit/s/Hz according to the methodology in [8], [15]. The average spectral efficiency $\bar{\gamma}$ is obtained by integrating over the cell area.

Table II contains the results for some representative parameter sets and Figure 7 shows graphs for the UMa scenario with incentives for voice traffic. UIL proposals (Table II) quantifies those locations where a move is recommended $\left(\gamma<\gamma_{\Theta}\right) . \bar{d}_{U I L}$ is the average distance from these locations to the nearest proposed point, if all users moved. The real

\begin{tabular}{|c|c|c|c|c|}
\hline Scenario & $\begin{array}{c}\text { Urban } \\
\text { micro } \\
\text { UMi }\end{array}$ & $\begin{array}{c}\text { Urban } \\
\text { macro } \\
\text { UMa }\end{array}$ & $\begin{array}{c}\text { Suburban } \\
\text { macro } \\
\text { SMa }\end{array}$ & $\begin{array}{c}\text { Rural } \\
\text { macro } \\
\text { RMa }\end{array}$ \\
\hline Inter-BS distance & $200 \mathrm{~m}$ & $500 \mathrm{~m}$ & $1299 \mathrm{~m}$ & $1732 \mathrm{~m}$ \\
\hline BS height & $10 \mathrm{~m}$ & $25 \mathrm{~m}$ & $35 \mathrm{~m}$ & $35 \mathrm{~m}$ \\
\hline Antenna tilt & $-12^{\circ}$ & $-12^{\circ}$ & $-6^{\circ}$ & $-6^{\circ}$ \\
\hline$f_{C}$ & $2.5 \mathrm{GHz}$ & $2.0 \mathrm{GHz}$ & $2.0 \mathrm{GHz}$ & $0.8 \mathrm{GHz}$ \\
\hline Tx power & $44 \mathrm{dBm}$ & $49 \mathrm{dBm}$ & $49 \mathrm{dBm}$ & $49 \mathrm{dBm}$ \\
\hline
\end{tabular}

TABLE II

SPECTRAL EFFICIENCY RESULTS $\bar{\gamma}$ IN [BIT/S/Hz/SECTOR] WITHOUT AND WITH UIL $\left(\gamma_{\Theta}=2.5 \mathrm{BIT} / \mathrm{s} / \mathrm{Hz}\right)$

\begin{tabular}{|c|c|c|c|c|}
\hline Scenario & UMi & UMa & SMa & RMa \\
\hline Without UIL [7]: $\bar{\gamma}=$ & 1.567 & 1.254 & 1.234 & 1.974 \\
\hline UIL proposals & $80 \%$ & $96 \%$ & $94 \%$ & $62 \%$ \\
\hline UIL $d_{U I L}=$ & $8.8 \mathrm{~m}$ & $9.4 \mathrm{~m}$ & $15.6 \mathrm{~m}$ & $61.4 \mathrm{~m}$ \\
\hline UIL with constant & 2.170 & 1.995 & 2.836 & 2.509 \\
$p_{M}=0.5 \rightarrow \bar{d}=$ & $4.4 \mathrm{~m}$ & $4.7 \mathrm{~m}$ & $7.8 \mathrm{~m}$ & $30.7 \mathrm{~m}$ \\
\hline UIL voice $\bar{\gamma}=$ & 2.319 & 2.293 & 3.030 & 2.170 \\
$i=-20 \% \rightarrow \bar{d}=$ & $4.5 \mathrm{~m}$ & $4.7 \mathrm{~m}$ & $7.3 \mathrm{~m}$ & $2.5 \mathrm{~m}$ \\
participation $p_{U I L}=$ & $69 \%$ & $74 \%$ & $55 \%$ & $22 \%$ \\
\hline UIL voice $\bar{\gamma} \overline{\bar{d}}=$ & 2.452 & 2.433 & 3.513 & 2.225 \\
$i=-40 \% \rightarrow \bar{d}=$ & $5.4 \mathrm{~m}$ & $5.4 \mathrm{~m}$ & $9.3 \mathrm{~m}$ & $3.3 \mathrm{~m}$ \\
participation $p_{U I L}=$ & $79 \%$ & $83 \%$ & $70 \%$ & $28 \%$ \\
\hline UIL voice $\bar{\gamma} \overline{\bar{d}}=$ & 2.588 & 2.535 & 3.941 & 2.254 \\
$i=-60 \% \rightarrow \bar{d}=$ & $6.7 \mathrm{~m}$ & $6.2 \mathrm{~m}$ & $11.0 \mathrm{~m}$ & $3.2 \mathrm{~m}$ \\
participation $p_{U I L}=$ & $88 \%$ & $89 \%$ & $83 \%$ & $31 \%$ \\
\hline UIL voice $\bar{\gamma}=$ & 2.595 & 2.554 & 3.924 & 2.385 \\
$i=-80 \% \rightarrow \bar{d}=$ & $7.0 \mathrm{~m}$ & $7.1 \mathrm{~m}$ & $11.9 \mathrm{~m}$ & $9.7 \mathrm{~m}$ \\
participation $p_{U I L}=$ & $88 \%$ & $90 \%$ & $83 \%$ & $43 \%$ \\
\hline UIL video $\bar{\gamma}=$ & 2.522 & 2.507 & 3.739 & 2.337 \\
$i=-40 \% \rightarrow \bar{d}=$ & $6.0 \mathrm{~m}$ & $6.2 \mathrm{~m}$ & $10.6 \mathrm{~m}$ & $8.7 \mathrm{~m}$ \\
participation $p_{U I L}=$ & $80 \%$ & $96 \%$ & $94 \%$ & $62 \%$ \\
\hline UIL data $\bar{\gamma}=$ & 2.969 & 2.995 & 3.577 & 2.156 \\
$I=$ speedup $\rightarrow \bar{d}=$ & $8.9 \mathrm{~m}$ & $9.4 \mathrm{~m}$ & $10.8 \mathrm{~m}$ & $6.4 \mathrm{~m}$ \\
participation $p_{U I L}=$ & $54 \%$ & $44 \%$ & $25 \%$ & $43 \%$ \\
\hline max gain for voice $\bar{\gamma}$ & $+66 \%$ & $+104 \%$ & $+218 \%$ & $+21 \%$ \\
\hline
\end{tabular}

participation $p_{U I L}$ can be observed as an outcome of all the user decisions taken at various positions and according to the surveyed behavior. In the UMi to SMa scenarios, even with 

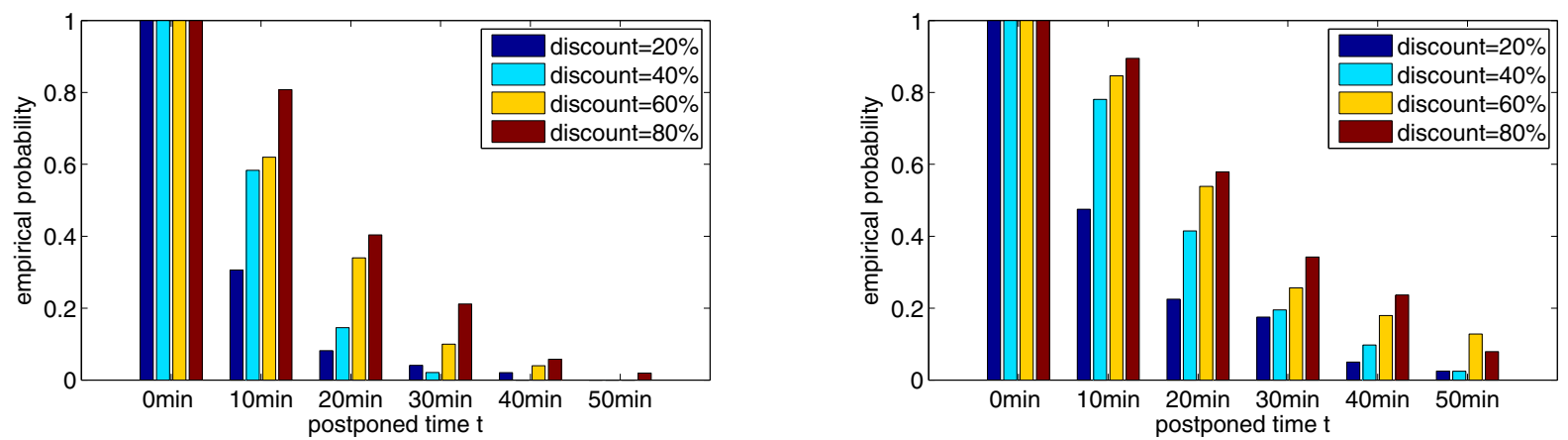

(a) Empirical CCDF for voice calls: With a given discount and a potential (b) Empirical CCDF for video streaming: With a given discount and a time $\mathrm{t}$ to postpone the session, how likely $\left(p_{M}\right)$ is the deferral of the user. potential time t to postpone the session, how likely $\left(p_{M}\right)$ is the deferral?
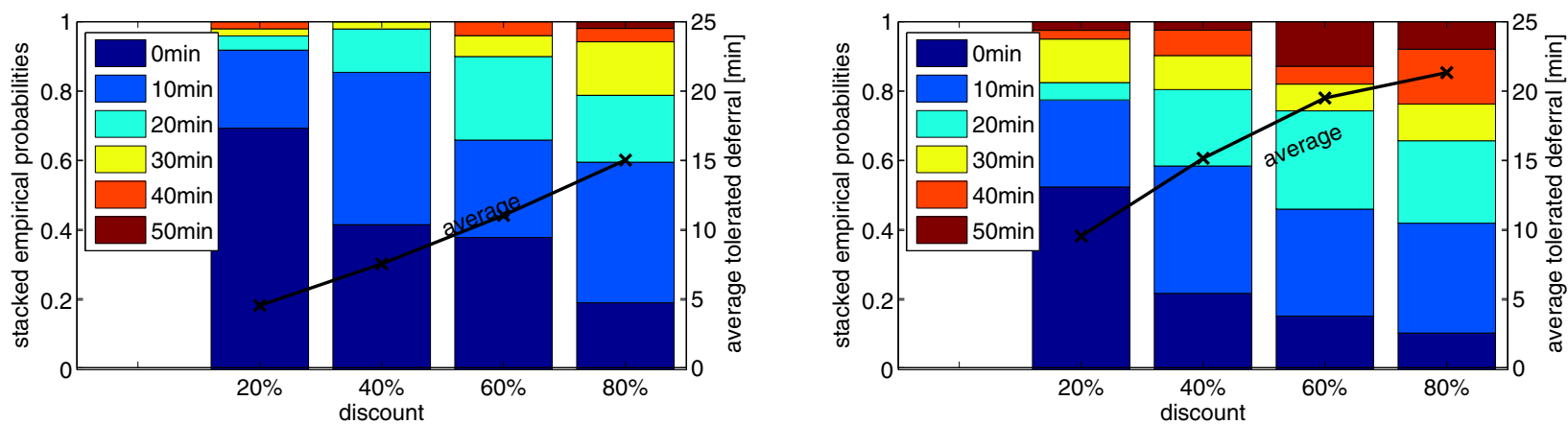

(c) For a given discount incentive for voice calls, what is the postponed (d) For a given discount incentive for video streaming, what is the session time distribution among all users and its average.

postponed session time distribution among all users and its average.

Fig. 5. Voice and video service comparison with temporal (postpone) control and discount incentives.

small incentive, more than $50 \%$ are motivated and with high incentive $p_{U I L}$ is within $[80 . .90 \%]$. The gains for $\bar{\gamma}$ are very encouraging. Especially the SMa scenario seems to benefit from the special geometry, such that for many positions there is likely a very high $\gamma$ nearby. Note that the huge gains allow more voice calls (Erlangs) in the busy hours so even with discount incentives of $-20 \%$ the total revenue is increased.

\section{CONCLUSION}

This paper supplements the new user-in-the-loop paradigm with a quantified user model based on survey results. It is an indicator for reactions in a real application scenario and improves upon the previous results based on assumptions only. UIL lets the user actively participate in improving the system performance. With an incentive (utility), either financial or higher data rate, there is a definite interest in participation. Numeric results obtained in a simulation study of IMT-Advanced scenarios show gains up to $200 \%$ independent of the PHY or MAC layer. The distances to move are easy to reach. The solution in this paper aims at sustainability (green) by reducing the wasteful use of limited resources. Therefore new telecom infrastructure needs to be deployed less frequently. The second effect is that users will be trained to understand why certain high-rate applications are prohibitive in the busy hours and at cell-edge locations, when the resource utilization is already $100 \%$. Green awareness is expected to motivate users even without explicit incentives (Figure 3), especially if long-term training is applied.

The sample size of this very first survey is small and results might not be as representative as a real-world field trial, but the UIL method is new and not yet out there. A sample Android app has been developed at Carleton University for the UIL concept and the results are encouraging.

\section{REFERENCES}

[1] "UMTS Forum report 44 - mobile traffic forecasts 2010-2020," http://www.umts-forum.org/, UMTS Forum, Tech. Rep., Jan 2011.

[2] "Cisco visual networking index: Global mobile data traffic forecast update, 2010-2015," Cisco Systems Inc., White Paper, February 1, 2011. [Online]. Available: http://www.cisco.com/en/US/solutions/

[3] "2010 mobile internet phenomena report," Sandvine Inc., White Paper. [Online]. Available: http://www.sandvine.com/downloads/documents/

[4] "Mobile broadband capacity constraints and the need for optimization," Rysavy Inc., White Paper, February 2010. [Online]. Available: http://www.rysavy.com/Articles/

[5] M. Dohler, R. Heath, A. Lozano, C. Papadias, and R. Valenzuela, "Is the PHY layer dead?" IEEE Communications Magazine, vol. 49, no. 4, pp. $159-165$, April 2011.

[6] J. Monserrat, P. Sroka, G. Auer, J. Cabrejas, D. Martin, A. Mihovska, R. Rossi, A. Saul, and R. Schoenen, "Advanced radio resource management for IMT-Advanced in WINNER+ (II)," in Proceedings of the ICT-MobileSummit 2010, Florence, Italy, Jun 2010.

[7] R. Schoenen, H. Yanikomeroglu, and B. Walke, "User-in-the-loop: Mobility aware users substantially boost spectral efficiency of cellular OFDMA systems," IEEE Communications Letters, vol. 15, no. 5, pp. 488-490, May 2011.

[8] R. Schoenen, "On increasing the spectral efficiency more than $100 \%$ by user-in-the-control-loop," in Proceedings of the 16th Asia-Pacific Conference on Communications (APCC), Auckland, October 2010.

[9] R. Schoenen, G. Bulu, A. Mirtaheri, and H. Yanikomeroglu, "Green communications by demand shaping and User-in-the-Loop tariff-based control," in Proceedings of the 2011 IEEE Online Green Communications Conference (IEEE GreenCom'11), Online, 2011.

[10] C. Courcoubetis, F. Kelly, V. Siris, and R. Weber, "A study of simple usagebased charging schemes for broadband networks," Telecommunication Systems, vol. 15, pp. 323-343, 2000.

[11] J. Altmann and K. Chu, "How to charge for network services - flat-rate or usage-based?" Computer Networks, vol. 36, no. 5-6, p. 519, 2001.

[12] L. A. DaSilva, "Pricing for QoS-enabled networks: A survey," IEEE Communications Surveys Tutorials, vol. 3, no. 2, pp. $2-8,2000$.

[13] R. Pabst, B. Walke, D. C. Schultz, H. Yanikomeroglu et al., "Relaybased deployment concepts for wireless and mobile broadband radio," IEEE Communications Magazine, pp. 80-89, Sep 2004.

[14] ITU, "Report ITU-R M2135; Guidelines for evaluation of radio interference technologies for IMT-Adcanced", 2008.

[15] R. Schoenen and B. Walke, "On PHY and MAC performance of 3GLTE in a multi-hop cellular environment," in Proceedings of the IEEE WiCom 2007, Shanghai, China, Sep 2007. 

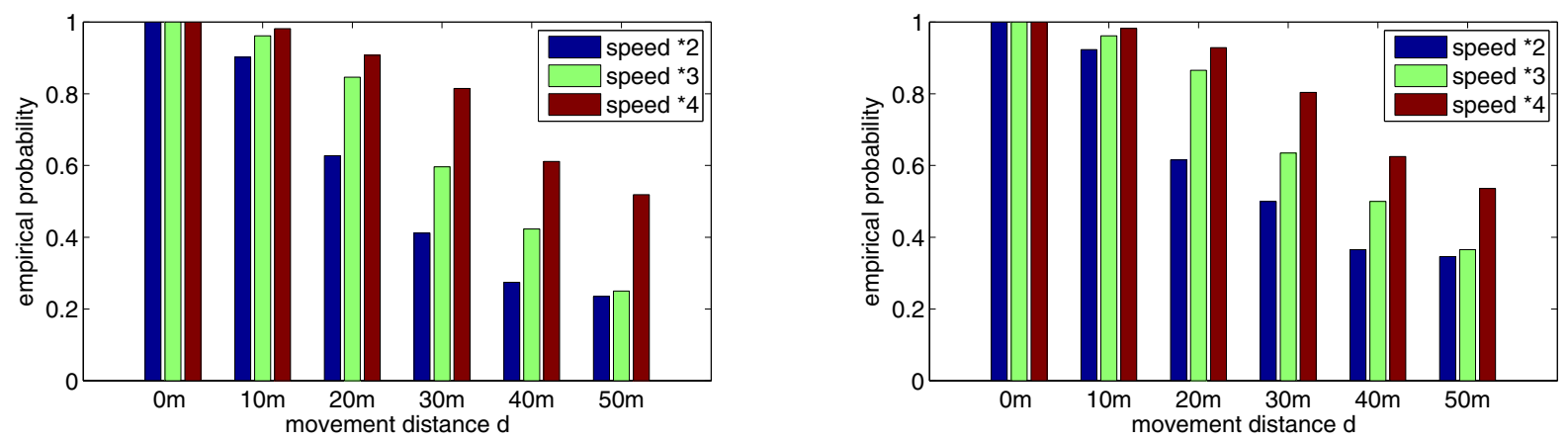

(a) Empirical CCDF for web browsing (data): With a given discount and (b) Empirical CCDF for app downloading (data): With a given discount a potential distance $d$ to move, how likely $\left(p_{M}\right)$ is the movement of the and a potential distance $d$ to move, how likely $\left(p_{M}\right)$ is the movement. user.
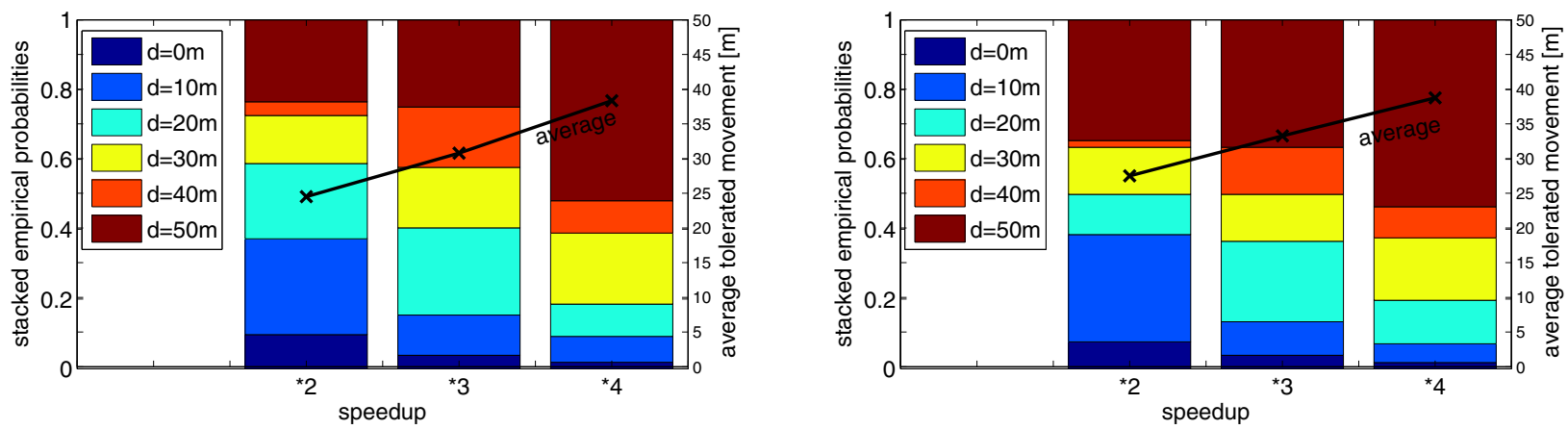

(c) For a given speedup incentive for web browsing, what is the tolerated (d) For a given speedup incentive for app downloading, what is the tolerated movement distribution among all users and its average. movement distribution among all users and its average.

Fig. 6. Data services (web browsing and app downloads) with movement control and speedup incentives.

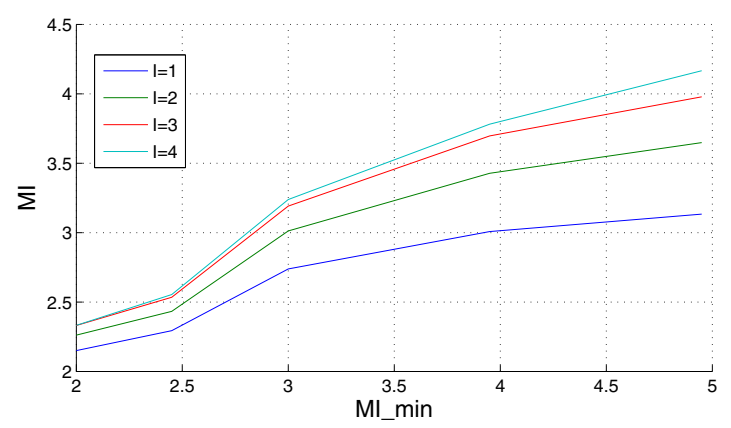

(a) Spectral efficiency in bit/s/Hz $\bar{\gamma}=f\left(\gamma_{\Theta}, I\right)$.

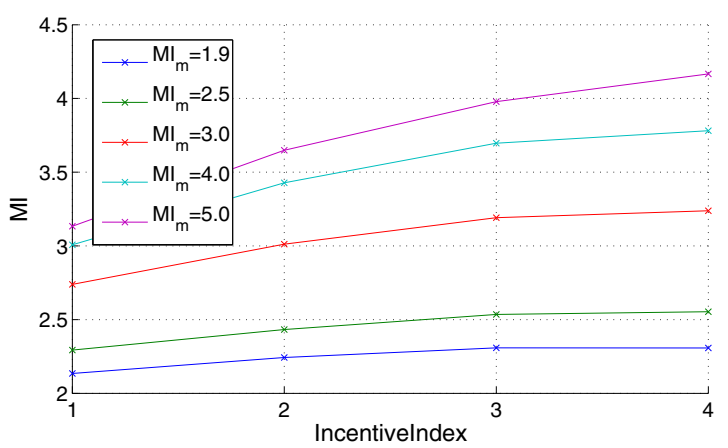

(c) Spectral efficiency in bit/s/Hz $\bar{\gamma}=f\left(I, \gamma_{\Theta}\right)$.

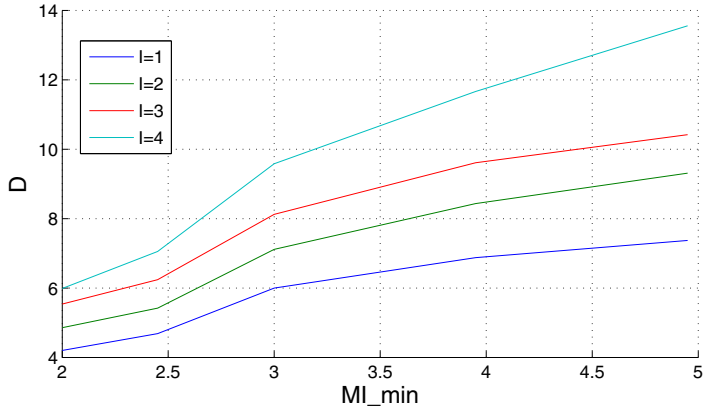

(b) Average move distance in meters $\bar{d}=f\left(\gamma_{\Theta}, I\right)$.

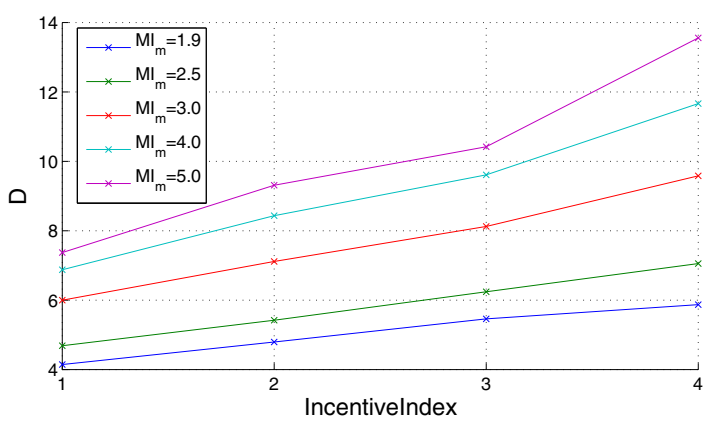

(d) Average move distance in meters $\bar{d}=f\left(I, \gamma_{\Theta}\right)$.

Fig. 7. Simulation results for LTE with UIL in IMT-Advanced UMa scenario. Incentive $I \in\{1,2,3,4\}$ corresponds to a voice call rate discount of $\{-20 \%,-40 \%,-60 \%,-80 \%\}$. The user behavior is assumed according to the survey results. Increasing the threshold $\gamma_{\Theta}$ and the incentive $I$ both lead to higher cell spectral effiency. This leads to the conclusion that telling the user all options (different $\gamma_{\Theta}$ ) is most beneficial. More powerful incentives are only required for $\gamma_{\Theta} \geq 3$ [7(c)]. Even with a small incentive discount $I_{1}=-20 \%, \bar{\gamma}$ can be increased from 1.254 (no UIL) to above 3 bits/s/Hz [7(a)], which economically more than compensates the impact of the incentive on the revenue in the busy hours. 